\title{
Implementation of Face Detection System using Adaptive Boosting Algorithm
}

\author{
Khizer Mehmood \\ Department of Electrical Engineering, \\ UET Taxila, Pakistan
}

\author{
Basit Ahmad \\ Department of Electrical Engineering, \\ UET Taxila, Pakistan
}

\begin{abstract}
Face detection is a very hot research topic in the fields of pattern recognition and computer vision. Its applications are widely used in artificial intelligence, surveillance video, identity authentication and human machine interaction. Face detection is based on identifying and locating a human face in the image, regardless of position, size, and condition. Various algorithms are proposed to detect faces in an image. This implementation is based on adaptive boosting algorithm and uses haar features which is based on statistical methods to detect face. Algorithm is implemented in MATLAB and synthesized by using Verilog on XILINX.
\end{abstract}

\section{Keywords}

Face Detection, Computer Vision, Adaboost

\section{INTRODUCTION}

Face detection is a very hot research topic in the fields of pattern recognition and computer vision. Its applications are widely used in artificial intelligence, surveillance video, identity authentication and human machine interaction. Face detection is based on identifying and locating a human face in the image, regardless of position, size, and condition. The goal of face detection is to determine whether or not there are any faces in the image and, if present, return the image location and extent of each face .Various challenges are associated with face detection. Those are pose, presence and absence of structural components, facial expression, occlusion, image orientation and image condition. Various techniques have been developed for face detection in single image. Generally they are categorized into four categories. Those are knowledge based methods [1], feature invariant approaches [2], template matching methods [3] and appearance based methods [4].

Knowledge based methods, feature invariant methods and template matching methods breaks down easily because of real world complexity. Among all these approaches appearance based methods are used because they rely on statistical analysis. Numerous approaches proposed in appearance based method namely eiganface [5], guassian distribution[6],neural network[7],support vector machine[8],naive bayes classifier[9],hidden markov model [10],information theoretical approach[11] and viola and jones based adaptive boosting algorithm[12].Among these algorithms adaptive boosting algorithm is widely used because it achieves fast, efficient and robust face detection. In their face detection implementation they have presented which is based on haar features. In haar features there are more than one scalar values which describes the average difference between two rectangular regions.
Several face detection algorithms along with their fpga implementations are presented in literature. Irick et al [13] presented a neural network based unified architecture for face detection applications. In their implementation they have used large pixel offset and apply to image size of $320 \times 240$ at $52 \mathrm{fps}$ and implemented on virtex-4 fpga board. Farrugia et al [14] presented a parallel optimized architecture using convolutional face finder (CFF) algorithm. They have applied the algorithm on image size of $320 \times 240$ at $29 \mathrm{fps}$ and implemented on virtex-4 fpga board. Goa and $\mathrm{Lu}$ [15] presented a novel architecture approach to accelerate the haar classifier for face detection applications. They have applied it on image size of $256 \times 192$ at 98fps and implemented on virtex-5 fpga board. JIN el al [16] presented face certainty map based face detection method. In face certainty map they have used large pyramid level, large haar like feature and small pixel offset which is scalable to frame rate and number of faces. They have applied it to an image size of 640X480 at $307 \mathrm{fps}$ and implemented on virtex-5 fpga board. He et al [17] proposed a novel system on chip architecture for ultra-fast face detection. They have used artificial neural network based classifiers using small pyramid level. They have used image size of $640 \times 480$ at $625 \mathrm{fps}$ and implemented on virtex-5 fpga board. Hiromoto el al [18] presented a hardware architecture based on adaboost learning algorithm. They have used image size of $640 \mathrm{X} 480$ at $30 \mathrm{fps}$ and implemented on virtex-5 fpga board. Ngo et al [19] presents an area efficient architecture for detection of multiple faces in video stream. They have used small haar like features and implemented for image size of $320 X 240$ at 30fps on Altera DE2 fpga board. Lai et al [20] presented architecture which uses small haar features and implemented for image size of $640 \mathrm{X} 480$ at $143 \mathrm{fps}$ on virtex-5 fpga board.

In this paper, hardware architecture for face detection in single image is proposed. Face Detection system creates a window is an integral part of the implementation of the haar classification feature. And then Performs parallel classification to detect the faces in image. This paper is organized as follows: In Section II, face detection algorithm is explained. In Section III, simulation results and analysis are presented. Conclusion is presented in Section IV.

\section{ALGORITHM FOR FACE DETECTION}

The block diagram of the algorithm is shown in Figure 1 


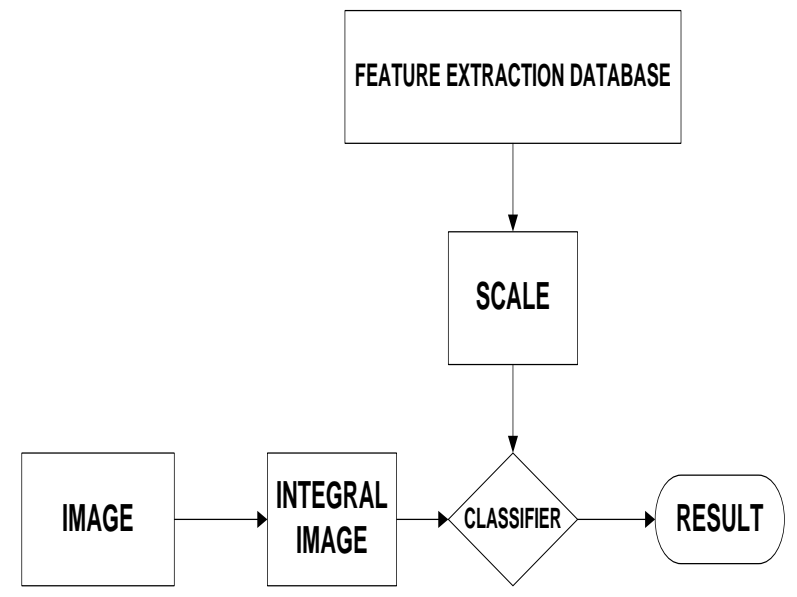

Fig 1: Block Diagram

The basic norm of the Adaboost algorithm is to scan a sub window proficient of finding faces across a given input image. Unlike in previous approaches the input image is rescaled and detector of fix size runs through these images which is time overwhelming due to calculation of dissimilar size images.

In adaboost algorithm instead of rescaling the size of image, size of detector is rescaled. By doing this the variable size detector scan the image. In order to avoid the calculation for the detector a new image representation is used so that the detector requires the same amount of calculations for any size of image. This representation is called integral image.

\subsection{Integral Image}

The major step of adaboost algorithm is to get the integral image representation of input image. This is done by assembly each pixel equivalent to the whole summation of all pixels above and to the leftward of the anxious pixel. Figure 2 shows the integral image representation.

\begin{tabular}{|c|c|c|}
\hline 1 & 1 & 1 \\
\hline 1 & 1 & 1 \\
\hline 1 & 1 & 1 \\
\hline
\end{tabular}

\begin{tabular}{|l|l|l|}
\hline \multicolumn{1}{c}{$\boldsymbol{1}$} & 2 & 3 \\
\hline 2 & 4 & 6 \\
\hline 3 & 6 & 9 \\
\hline
\end{tabular}

Fig 2: Integral Image Representation

\subsection{Feature Extraction}

The adaboost centered face detection technique uses a set of modest rectangular features to spot faces. Various types of rectangles are employed which are shown in Figure 3.

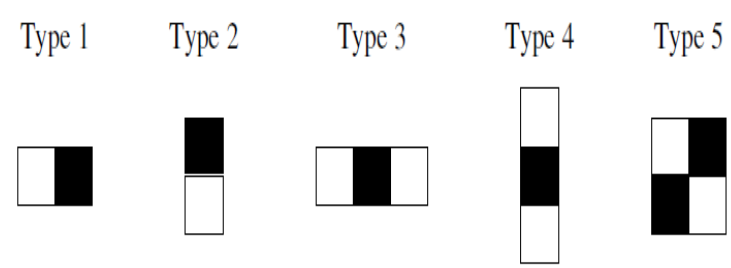

Fig 3:Types of Haar Features

\subsection{Scaling}

Scaling is required in most of the cases because size of face is variable in an image. In order to spot all the faces in an image the scaler module is used to resize the sub frame. It supports nearest neighbor, bilinear and poly-phase scaling algorithms. In all these algorithms nearest neighbor is used because it consumes less hardware resources.

\subsection{Classifier}

Classifier uses the rectangle integral to calculate the value of a feature. Classifier are used in cascade architecture because if the image contain more than one face than it takes large amount of time to evaluate sub windows. The cascaded classifier is composed of stages each containing a strong classifier. The job of each stage is to determine whether a given sub window is definitely not a face or may be a face. When a sub window is classified to be a non-face by a given stage it is immediately discarded. Conversely a sub window classified as a may be face is passed on to the next stage in the cascade. Figure 4 shows the cascade architecture.

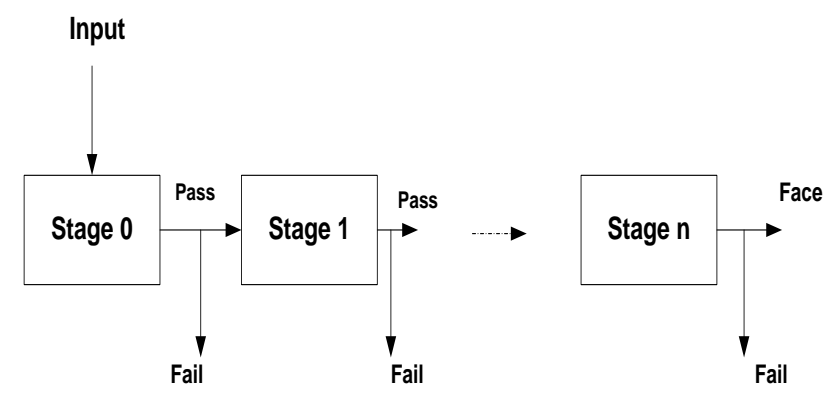

Fig 4: Classifier

\subsection{Haar Features}

There are numerous scales, positions and kinds for haar features. There structure and scheme is simple. Here eigenvalues are addition of black area pixels subtracts the sum of white area pixels in the rectangle. It allows very well grained review of files, it also rises the training time and can decrease the performance of the circular. For these reasons, the approach Viola Jones algorithm uses Adaboost promotion as a means to select feature by creating a weak workbook for every haar feature. Specifically, binary workbook is created on the basis of threshold each Haar feature so error is reduced training likely. During every round of the increase, one is chosen best workbook weak for that round (consistent to a special feature Haar). The final effect of the increase is a powerful seed production is calculated as a combination of a weak linear threshold works. Viola Jones based face detection method is both fast and efficient. The architecture for Haar feature classifier is shown in figure 5 . 


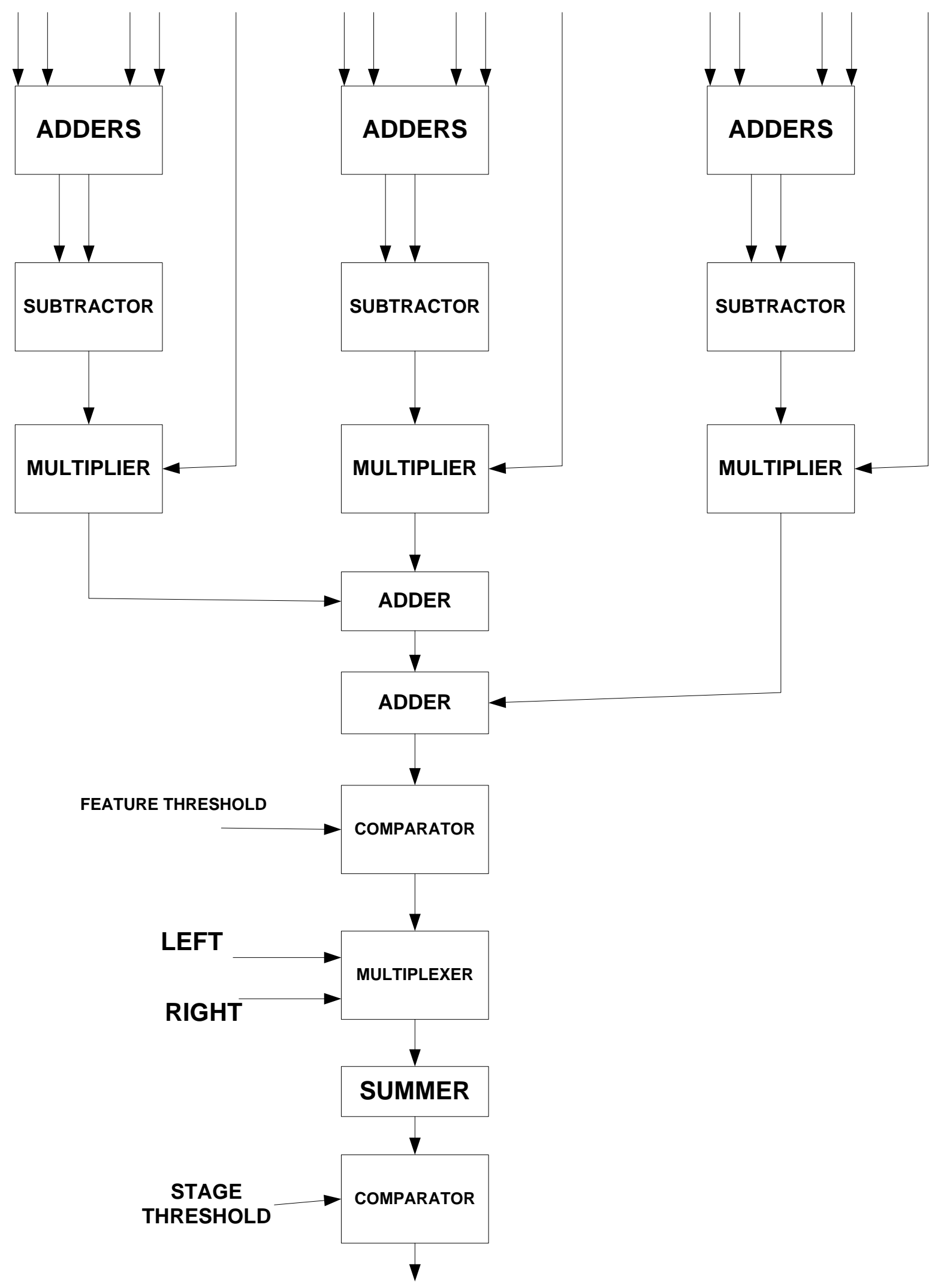

Fig 5: Haar Classifier Architecture 


\section{IMPLEMENTATION AND RESULTS}

The simulation is in two steps. First the input image is converted into gray scale and its integral image representation is calculated. Then the input image is given to the modal. The modal detects the face by using haar features of adaptive boosting algorithm. We have used AR face database [21] for testing of algorithm. It includes 252 images. We have arbitrarily select $85 \%$ of images as training set and $15 \%$ as testing set. The algorithm is tested on MATLAB. We have divided the images into three categories. Those are:

- $\quad$ Single frontal faces

- Near frontal multiple faces

- Multiple faces with different size occlusion

\subsection{Single Frontal Face}

In single frontal faces there is no occlusion. Results are shown in figure $6(\mathrm{a}, \mathrm{b}, \mathrm{c})$

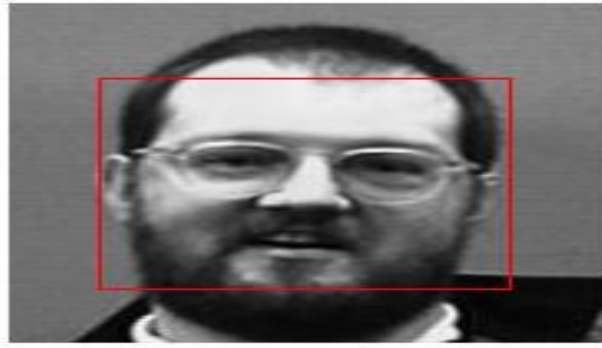

(a)

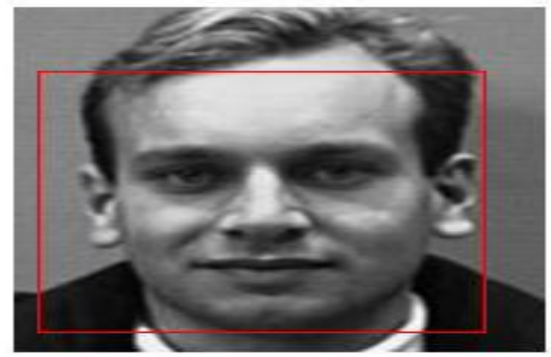

(b)

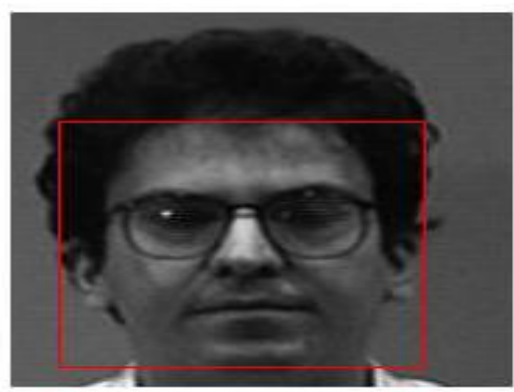

(c)

Fig 6: Single frontal Images
Table 1. Single Frontal Images

\begin{tabular}{|c|c|c|c|c|c|}
\hline $\begin{array}{c}\text { Image } \\
\text { S }\end{array}$ & Size & $\begin{array}{c}\text { Total } \\
\text { Faces }\end{array}$ & $\begin{array}{c}\text { False } \\
\text { Negative }\end{array}$ & $\begin{array}{c}\text { False } \\
\text { Positive }\end{array}$ & $\begin{array}{c}\text { Face } \\
\text { Detected }\end{array}$ \\
\hline 1 & $200 \times 180$ & 1 & 0 & 0 & 1 \\
\hline 2 & $200 \times 180$ & 1 & 0 & 0 & 1 \\
\hline 3 & $200 \times 180$ & 1 & 0 & 0 & 1 \\
\hline
\end{tabular}

\subsection{Near Frontal Multiple Faces}

In near frontal multiple face images. There are multiple faces in an image and all these faces have different size occlusion. Results are shown in figure $7(\mathrm{a}, \mathrm{b}, \mathrm{c})$.

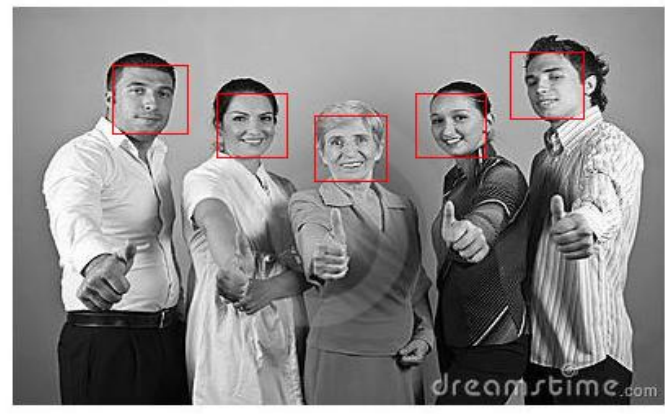

(a)

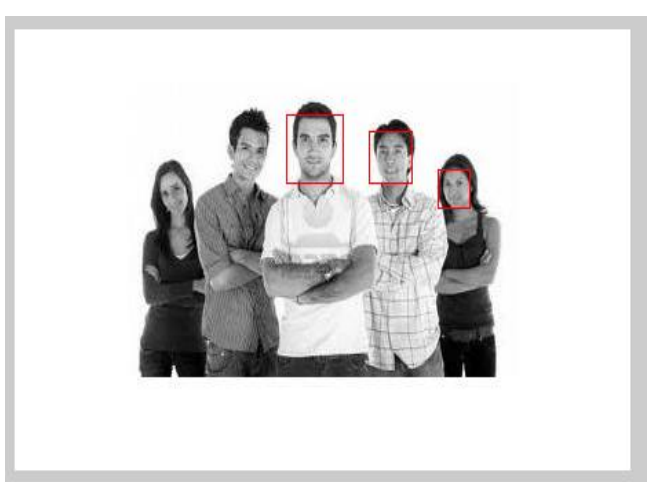

(b)

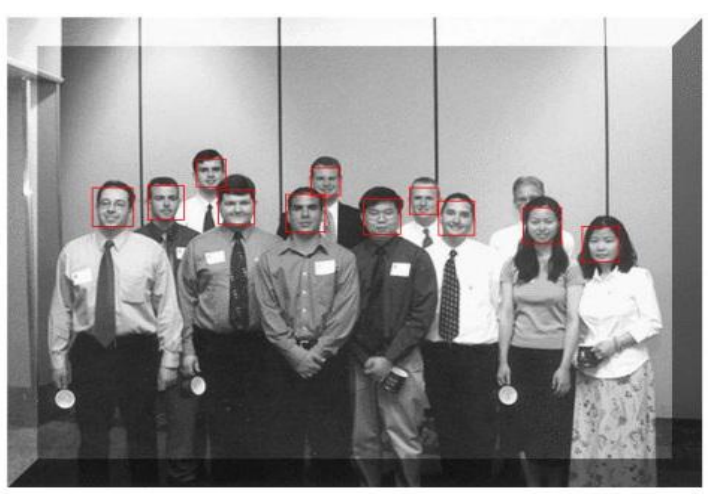

(c)

Fig 7: Multiple Near frontal Images 
Table 2. Multiple Near Frontal Images

\begin{tabular}{|c|c|c|c|c|c|}
\hline $\begin{array}{c}\text { Image } \\
\text { s }\end{array}$ & Size & $\begin{array}{c}\text { Total } \\
\text { Faces }\end{array}$ & $\begin{array}{c}\text { False } \\
\text { Negative }\end{array}$ & $\begin{array}{c}\text { False } \\
\text { Positive }\end{array}$ & $\begin{array}{c}\text { Face } \\
\text { Detected }\end{array}$ \\
\hline 1 & $565 \times 354$ & 5 & 0 & 0 & 5 \\
\hline 2 & $447 \times 265$ & 5 & 2 & 0 & 3 \\
\hline 3 & $764 \times 512$ & 12 & 1 & 0 & 11 \\
\hline
\end{tabular}

\subsection{Multiple Faces with different Size Occlusion}

In multiple faces with different size occlusion there are multiple faces in images with different size occlusion and different level of rotation. Results are shown in figure $8(a, b$, c).

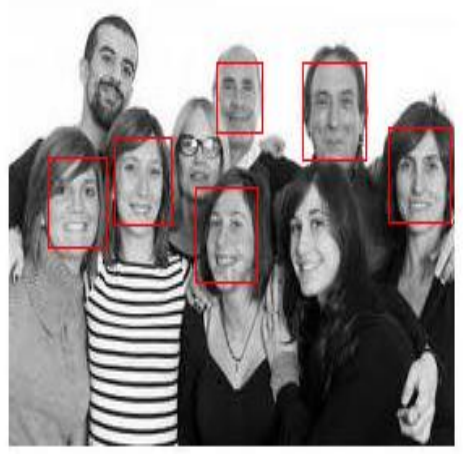

(a)

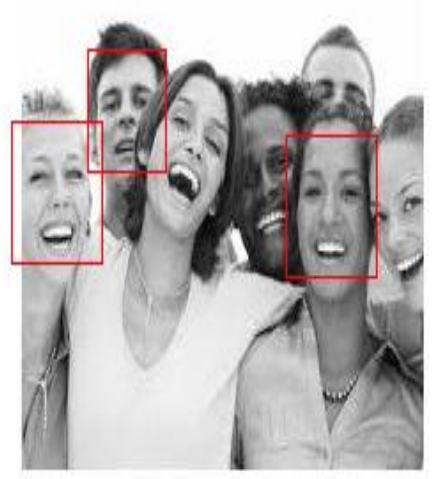

(b)

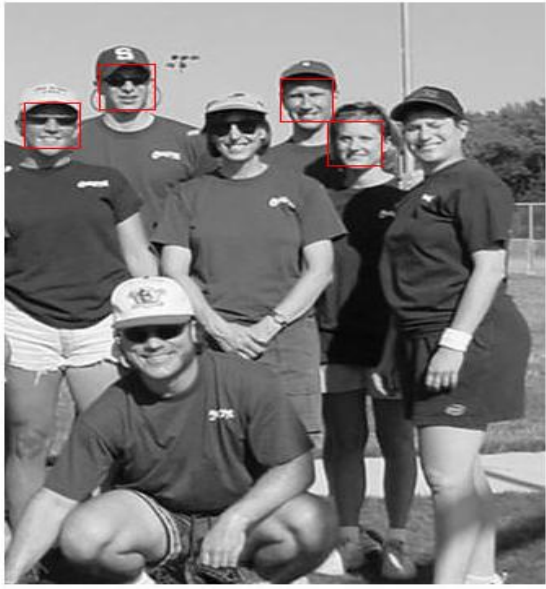

(c)

Fig 8: Multiple Faces with different Size Occlusion

Table 3. Multiple Faces with different Size Occlusion

\begin{tabular}{|c|c|c|c|c|c|}
\hline $\begin{array}{c}\text { Image } \\
\text { s }\end{array}$ & Size & $\begin{array}{c}\text { Total } \\
\text { Faces }\end{array}$ & $\begin{array}{c}\text { False } \\
\text { Negative }\end{array}$ & $\begin{array}{c}\text { False } \\
\text { Positive }\end{array}$ & $\begin{array}{c}\text { Face } \\
\text { Detected }\end{array}$ \\
\hline 1 & $439 \times 269$ & 9 & 3 & 0 & 6 \\
\hline 2 & $391 \times 309$ & 7 & 4 & 0 & 3 \\
\hline 3 & $496 \times 522$ & 7 & 3 & 0 & 4 \\
\hline
\end{tabular}

The algorithm is implemented by using Verilog HDL on XILINX. There are two implementations. In first implementation there are integral representations of face images while in the second representation there are non-face images. In Verilog implementation to setup the integral image a 17-bit input is used. In order to read the address of integral image two 5-bit inputs are used. As integral image is in separate module which is being instantiated later it is stored in register. For reading the value of integral image four 12 registers each of 17-bit are used. For checking the threshold at each stage check points are used. An FSM is used to check whether a stage is failed or face is detected. Conditionals are employed on output signals to temporarily store the final output. The RTL schematic is shown in figure 9.

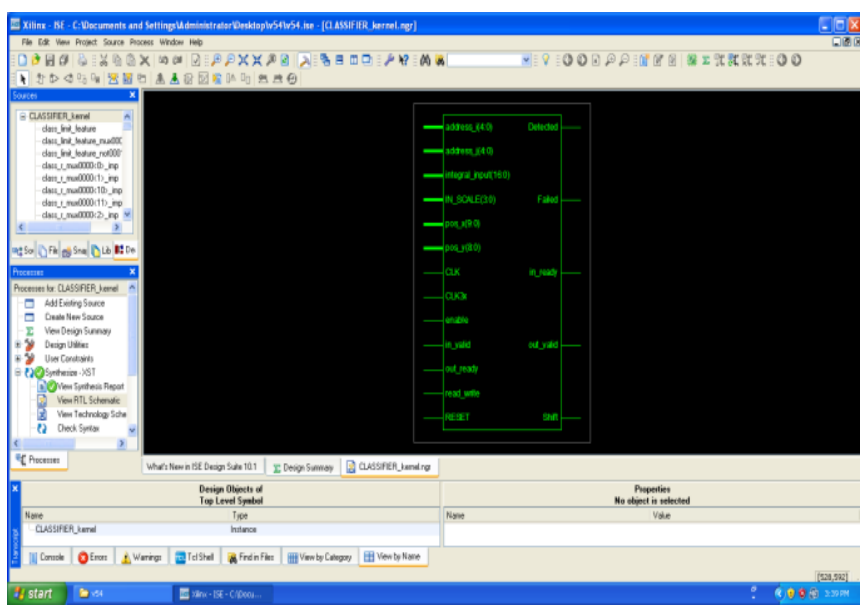

Fig 9: RTL Schematic 
Table 4. Resource Utilization Summary

\begin{tabular}{|c|c|c|c|}
\hline Resources & Available & Used & Utilization \\
\hline Registers & 19200 & 7431 & $38 \%$ \\
\hline LUTs & 19200 & 14251 & $74 \%$ \\
\hline Block RAM & 220 & 59 & $26 \%$ \\
\hline DSP48Es & 32 & 3 & $9 \%$ \\
\hline
\end{tabular}

Table 5. Synthesis Summary

\begin{tabular}{|c|c|}
\hline $\begin{array}{c}\text { ROM } \\
22 \text { X8 bit }\end{array}$ & 2 \\
\hline MACs & \\
\hline 17x3-to-21-bit MAC & 1 \\
\hline 17x3-to-22-bit MAC & 1 \\
\hline Multipliers & \\
\hline 17x3-bit multiplier & 1 \\
\hline Adders/Subtractors & 20 \\
\hline 12-bit adder & 3 \\
\hline 17-bit adder & 3 \\
\hline 17-bit subtractor & 6 \\
\hline 19-bit adder & 1 \\
\hline 5-bit adder & 3 \\
\hline 5-bit adder carry out & 2 \\
\hline 6-bit subtractor & 2 \\
\hline Registers & 7431 \\
\hline Flip-Flops & 7431 \\
\hline Comparators & 13 \\
\hline 10-bit comparator greater & 3 \\
\hline 10-bit comparator less & 3 \\
\hline 12-bit comparator equal & 2 \\
\hline 19-bit comparator greater & 1 \\
\hline 26-bit comparator less & 1 \\
\hline 4-bit comparator less & 1 \\
\hline 9-bit comparator greater & 1 \\
\hline 9-bit comparator less & 1 \\
\hline
\end{tabular}

\section{CONCLUSION}

The main objective of this work was to design and implementation of face detection system. A Face Detection system is designed and implemented using adaptive boosting algorithm. It is implemented on MATLAB and XILINX. In MATLAB three cases of faces are considered and error is calculated on the basis of false positives and false negatives. Finally verilog implementation is synthesized by using XILINX.

\section{REFERENCES}

[1] G. Yang and T. S. Huang, "Human Face Detection in Complex Background," Pattern Recognition, vol. 27, no. 1, pp. 53-63, 1994.

[2] K.C. Yow and R. Cipolla, "A Probabilistic Framework for Perceptual Grouping of Features for Human Face Detection," Proc. Second Int'l Conf. Automatic Face and Gesture Recognition, pp. 16-21, 1996.

[3] A. Lanitis, C.J. Taylor, and T.F. Cootes, "An Automatic Face Identification System Using Flexible Appearance Models," Image and Vision Computing, vol. 13, no. 5, pp. 393-401, 1995.

[4] K.-K. Sung and T. Poggio, "Example-Based Learning for View- Based Human Face Detection," IEEE Trans. Pattern Analysis andMachine Intelligence, vol. 20, no. 1, pp. 39-51, Jan. 1998.

[5] M. Turk and A. Pentland, "Eigenfaces for Recognition," J. Cognitive Neuroscience, vol. 3, no. 1, pp. 71-86, 1991

[6] K.-K. Sung and T. Poggio, "Example-Based Learning for View-Based Human Face Detection," IEEE Trans. Pattern Analysis and Machine Intelligence, vol. 20, no. 1, pp. 39-51, Jan. 1998.

[7] H. Rowley, S. Baluja, and T. Kanade, "Neural NetworkBased Face Detection," IEEE Trans. Pattern Analysis and Machine Intelligence,vol. 20, no. 1, pp. 23-38, Jan. 1998.

[8] E. Osuna, R. Freund, and F. Girosi, "Training Support Vector Machines: An Application to Face Detection," Proc. IEEE Conf.Computer Vision and Pattern Recognition, pp. 130-136, 1997.

[9] H. Schneiderman and T. Kanade, "Probabilistic Modeling of Local Appearance and Spatial Relationships for Object Recognition,'Proc. IEEE Conf. Computer Vision and Pattern Recognition, pp. 45-51,1998.

[10] A. Rajagopalan, K. Kumar, J. Karlekar, R. Manivasakan, M. Patil, U. Desai, P. Poonacha, and S. Chaudhuri, "Finding Faces in Photographs," Proc. Sixth IEEE Int'1 Conf. Computer Vision, pp. 640-645, 1998.

[11] M.S. Lew, "Information Theoretic View-Based and Modular Face Detection," Proc. Second Int'l Conf. Automatic Face and Gesture Recognition, pp. 198-203, 1996.

[12] P. Viola and M. Jones, "Robust real-time object detection," International Journal of Computer Vision, 57(2), 137-154, 2004.

[13] K. Irick, M. DeBole, V. Narayanan, R. Sharma, H. Moon, and S. Mummareddy, "A unified streaming architecture for real time face detection and gender classification," in Proc. Int. Conf. Field Programmable Logic Appl., Aug. 2007, pp. 267-272

[14] N. Farrugia, F. Mamalet, S. Roux, F. Yang, and M. Paindvoine, "Fast and robust face detection on a parallel optimized architecture implemented on FPGA," IEEE 
Trans. Circuits Syst. Video Technol., vol. 19, no. 4, pp. 597-602, Apr. 2009.

[15] C. Gao and S. Lu, "Novel FPGA based Haar classifier face detection algorithm acceleration," in Proc. Int. Conf. Field Programm. Logic Appl., Sep. 2008, pp. 373-378.

[16] Seunghun Jin; Dongkyun Kim; Thuy Tuong Nguyen; Daijin Kim; Munsang Kim; Jae Wook Jeon; , "Design and Implementation of a Pipelined Datapath for HighSpeed Face Detection Using FPGA," Industrial Informatics, IEEE Transactions on , vol.8, no.1, pp.158167, Feb. 2012

[17] C. He, A. Papakonstantinou, and D. Chen, "A novel SoC architec-ture on FPGA for ultra-fast face detection," in Proc. IEEE Int. Conf. Comput. Design, Oct. 2009, pp. $412-418$
[18] M. Hiromoto, K. Nakahara, and H. Sugano, "A specialized processor suitable for AdaBoost-based detection with Haar-like features," in Proc. IEEE Conf. Comput. Vis. Pattern Recognit., Oct. 2007, pp. 1-8.

[19] H. Ngo, R. Tompkins, J. Foytik, and V. Asari, "An area efficient modular architecture for real-time detection of multiple faces in video stream," in Proc. 6th Int. Conf. Inf., Commun. Signal Process., 2007,pp. 1-5.

[20] H.-C. Lai, M. Savvides, and T. Chen, "Proposed FPGA hardware architecture for high frame rate (>100 fps) face detection using featurecascade classifiers," in Proc. IEEE Int. Conf. Biometr.: Theory, Appl., Syst., Sep. 2007, pp. 1-6.

[21] "The Face Database from Ohio State University." [Online].Available: http://www2.ece.ohiostate.edu/ aleix/ARdatabase.html 